\title{
Ocorrência de Salmonella sp em suínos de terminação no Rio Grande do Sul ${ }^{1}$
}

\author{
Lúcia H. N. Weiss ${ }^{2}$, Ricardo B. Nonig ${ }^{3}$, Marisa Cardoso ${ }^{4 *}$ e Marisa da Costa ${ }^{5}$
}

\begin{abstract}
Weiss L.H.N., Nonig R., Cardoso M. \& Costa M. 2002. [Occurrence of Salmonella sp in finishing pigs in Rio Grande do Sul, Brazil.] Ocorrência de Salmonella sp em suínos de terminação no Rio Grande do Sul. Pesquisa Veterinária Brasileira 22(3):104-108. Depto Medicina Veterinária Preventiva, Faculdade de Veterinária, UFRGS, Av. Bento Gonçalves 9090, Porto Alegre, RS 91540-000, Brazil.

Pen feces samples were taken in ten finishing farms in Rio Grande do Sul (Brazil) and submitted to Salmonella isolation protocol. Two farms negative and one positive in the preliminary screening were chosen, and feces from 25 randomly selected pigs in each of them were individually collected. The same animals were later sampled (rectal swab, intestinal content and mesenteric lymph nodes) at the slaughterhouse. After the introduction of new animals into farm $\mathrm{N} 1$ and $\mathrm{P} 1$, other 25 pigs on each farm were examined as described above. In the preliminary screening, Salmonella was isolated from pen feces samples of 3 of the 10 investigated farms. Two of these herds showed a high level contamination. Eight Salmonella serotypes (Agona, Bredeney, Lexington, London, Mbandaka, Panama, Schwartzengrund, Salmonella sp) were found, with serotypes Agona and Bredeney being the most frequent. When individual animals were sampled, Salmonella was isolated in all selected farms. Salmonella was isolated in $6.4 \%$ of feces collected on the farm, $5.3 \%$ of intestinal contents and $5.6 \%$ of lymph nodes. Antibiogram testing of the isolated strains showed $97.8 \%$ resistance to sulphonamides, $82.6 \%$ to streptomycin, $36.9 \%$ to tetracyclin and $15.2 \%$ to sulfazotrim.
\end{abstract}

INDEX TERMS: Salmonella, swine, lymph nodes, feces, serotypes, antibiogram.

RESUMO.- Foram colhidas amostras de fezes de lote de suínos em 10 granjas terminadoras do Estado do Rio Grande do Sul para pesquisa de Salmonella sp. Duas granjas consideradas negativas ( $\mathrm{N} 1$ e N2) e uma positiva ( $\mathrm{P} 1$ ) nesta primeira etapa foram escolhidas para colheita de amostras de fezes individuais de 25 animais escolhidos aleatoriamente. No abatedouro foram coletados swab retal, conteúdo intestinal e linfonodos mesentéricos dos mesmos animais amostrados na granja. Após a introdução de novos animais nas granjas N1 e P1, outros 25 animais foram amostrados em cada granja, da mesma forma descrita acima. Três granjas tiveram amostras de fezes de lote positivas, sendo que em duas foi consta-

\footnotetext{
${ }^{1}$ Aceito para publicação em 29 de agosto de 2001.

${ }^{2}$ Mestre em Ciências Veterinárias, UFRGS.

${ }^{3}$ Mestre em Microbiologia Agrícola e do Ambiente, UFRGS.

${ }^{4}$ Depto Medicina Veterinária Preventiva, Faculdade de Veterinária, UFRGS, Av. Bento Gonçalves 9090, Porto Alegre, RS 91540-000. *Autor para correspondência.

${ }^{5}$ Depto Microbiologia, Instituto de Ciências Básicas da Saúde, UFRGS.
}

tado um alto nível de contaminação. Foram encontrados 8 sorotipos de Salmonella (Agona, Bredeney, Lexington, London, Mbandaka, Panama, Schwartzengrund, Salmonella sp), sendo os sorotipos Agona e Bredeney os mais encontrados. Na colheita individual realizada, todas as granjas amostradas foram positivas. Em $6,4 \%$ das amostras de fezes colhidas na granja, $5,3 \%$ das amostras de conteúdo intestinal e 5,6\% dos linfonodos mesentéricos foi possível isolar Salmonella. O antibiograma das linhagens de Salmonella isoladas demonstrou $97,8 \%$ de resistência à sulfonamida, $82,6 \%$ à estreptomicina, $36,9 \%$ à tetraciclina e $15,2 \%$ à sulfazotrim.

TERMOS DE INDEXAÇÃO: Salmonella, suínos, fezes, linfonodos, sorotipos, antibiograma.

Classificação CNPq: 5.05.02.00-0 Medicina Veterinária Preventiva

\section{INTRODUÇÃO}

A salmonelose é uma zoonose de importância mundial. A ampla distribuição do gênero Salmonella entre os animais e sua permanência no ambiente contribuem para que este microrganismo assuma um papel importante na saúde pública. 
Alguns sorotipos do gênero Salmonella apresentam maior especificidade para determinados hospedeiros, enquanto outros afetam um grande número de espécies animais (Hirsh 1990). Em suínos poucos sorotipos são causa de doença clínica, entretanto aqueles que não estão associados a esses quadros são os principais envolvidos na contaminação da carne suína e seus produtos (Fedorka-Cray \& Gray 1996). Desta forma, a epidemiologia da salmonelose em suínos deve ser observada como dois problemas distintos: a salmonelose como infeção clínica e a contaminação por Salmonella em carcaças suínas e seus produtos. Relatos sobre o isolamento de Salmonella em produtos suínos têm pouca relação com a prevalência da doença em suínos. (Wilcock \& Schwartz 1993).

Embora a Salmonella possa sobreviver por longos períodos no ambiente, é amplamente aceito que os animais portadores são a maior fonte de infeção para animais e humanos (Wray \& Sojka 1977). O estresse pode ser um importante fator na reativação de suínos portadores assintomáticos que não estão excretando Salmonella (Williams \& Newell 1970). Estudos têm demonstrado o aumento da taxa de excreção de espécies de Salmonella após o estresse de transporte da granja produtora até o abatedouro (Williams \& Newell 1970, Berends et al. 1996), tornando estes animais um perigo em potencial para a segurança alimentar.

No Brasil os relatos de isolamento de Salmonella em granjas produtoras de suínos estão associados ao quadro clínico da doença, tanto na forma entérica como septicêmica (Langenegger \& Langenegger 1975, Barcellos et al. 1980, Ferraz et al. 1984, Barcellos et al. 1984). Em animais clinicamente normais tem sido encontrado diferentes níveis de portadores em linfonodos colhidos no momento do abate (Neiva 1946, Costa et al. 1972, Langenegger et al. 1983, Alves et al. 1994).

Nos últimos anos os produtos de origem suína ganharam atenção como fontes potenciais de salmonelose em humanos, principalmente depois que surtos de toxinfecção alimentar tiveram sua origem associada ao consumo destes produtos na Dinamarca (Fedorka-Cray 1996). Após a implantação do programa dinamarquês de controle de salmonelose, outros importantes países produtores de suínos iniciaram estudos de prevalência de Salmonella e tomaram medidas para seu controle nos rebanhos, como forma de garantir a qualidade dos produtos oferecidos no mercado interno ou externo (Davies \& Funk 1999).

Para alcançar competitividade no mercado externo, face à exigência crescente dos consumidores no sentido de melhoria no padrão sanitário dos produtos de origem animal, o Brasil necessita seguir o exemplo dos outros países produtores e iniciar programas de controle de Salmonella em suínos.

Este trabalho teve como objetivo realizar um estudo inicial da ocorrência de Salmonella em suínos de terminação no Rio Grande do Sul, comparando o nível de isolamento durante a permanência dos animais nas granjas e a partir de fezes e linfonodos colhidos dos mesmos animais no abatedouro.

\section{MATERIAL E MÉTODOS}

Seleção inicial. Em 10 granjas terminadoras de suínos do Rio Grande do Sul foram colhidas amostras de fezes de lote para pesquisa de Salmonella. Entende-se por fezes de lote a mistura de fezes de vários animais, colhidas do piso de seis locais de uma baia. Em cada granja foram colhidas 10 amostras de fezes de lote em diferentes baias, totalizando 100 amostras. As amostras foram mantidas sob refrigeração até o processamento no laboratório. Concomitantemente, foi feita a colheita de duas amostras de ração em cada uma das granjas visitadas.

Primeira colheita. Duas granjas (N1 e N2) sem isolamento de Salmonella e uma granja positiva (P1) na seleção inicial foram escolhidas. A escolha das granjas negativas foi feita de acordo com o calendário previsto para o abate dos animais. A granja positiva foi escolhida pelo mesmo critério, dentre aquelas que apresentaram um alto nível de isolamento de Salmonella durante a seleção inicial.

Em cada uma das granjas foram escolhidos 25 animais de forma aleatória, sendo colhidas fezes individualmente do reto de cada animal. Após a colheita, os animais foram marcados para posterior reconhecimento no abatedouro. Em cada granja foram ainda colhidas duas amostras de ração.

No abatedouro, os mesmos animais que haviam sido marcados na granja foram alojados em uma baia separada e a colheita realizada em duas etapas: antes do abate e na linha de processamento. Antes do abate foram colhidas, de cada um dos animais, swabs retais que foram imediatamente colocados em tubos contendo $9 \mathrm{ml}$ de água peptonada tamponada. Na linha de abate, após a evisceração, foram colhidos linfonodos mesentéricos e um segmento do intestino próximo ao ceco para análise do seu conteúdo.

Segunda colheita. Após a introdução de novos animais nas granjas N1 e P1, outros 25 animais de cada uma das granjas foram amostrados individualmente como descrito na primeira colheita.

Processamento das amostras. Cada amostra de ração, fezes de lote, linfonodos e conteúdo intestinal foi processada separadamente. De cada amostra foram pesados $25 \mathrm{~g}$ em sacos estéreis, adicionados de água peptonada tamponada e homogeneizados em Stomacher. A mistura foi então transferida para um frasco Erlenmeyer estéril, completada até $250 \mathrm{ml}$ com água peptonada tamponada e incubados a $37^{\circ} \mathrm{C}$ por $18 \mathrm{~h}$ (etapa de pré-enriquecimento). Após a incubação alíquotas de $0,1 \mathrm{ml}$ ou 1 $\mathrm{ml}$ foram semeadas, respectivamente, em Caldo RappaportVassiliadis (RV, Merck) e Caldo Tetrationato Müller-Kaufmann (TMK, Difco). Após incubação a $42^{\circ} \mathrm{C}$ (caldo RV) ou $37^{\circ} \mathrm{C}$ (caldo TMK), alíquotas de ambos os meios foram semeadas em ágar XLT4 (Difco) e ágar Rambach (Merck). Colônias suspeitas foram identificadas por testes bioquímicos, confirmadas com soro polivalente somático anti-Salmonela (Probac) e enviadas para sorotipagem no Instituto Oswaldo Cruz, Rio de Janeiro, RJ.

As amostras de swab retal foram incubadas nos tubos com água peptonada tamponada onde haviam sido colocados no momento da colheita por $18 \mathrm{~h} \mathrm{a} 37^{\circ} \mathrm{C}$ e posteriormente processados como descrito acima.

Antibiograma. Todas as amostras de Salmonella sp foram testadas quanto à sensibilidade a antimicrobianos pelo método da difusão em ágar descrito por Barry \& Thornsberry (1985) com discos de diversos antimicrobianos (Cecon). Foram testados: ácido nalidíxico $(30 \mathrm{mg})$, amoxacilina/ácido clavulânico $(10 \mathrm{mg})$, ampicilina $(10 \mathrm{mg})$, cefalotina $(30 \mathrm{mg})$, cloranfenicol $(30 \mathrm{mg})$, espectinomicina (10 $\mathrm{mg})$, estreptomicina $(10 \mathrm{mg})$, gentamicina (10 $\mathrm{mg}$ ), neomicina (30 mg), sulfonamida (300 mg), sulfazotrim (30 mg) e tetraciclina $(30 \mathrm{mg})$. 


\section{RESULTADOS E DISCUSSÃO}

A colheita de fezes de lote tem sido a metodologia mais utilizada para determinar a presença de animais portadores de Salmonella nas granjas de criação de suínos (Fedorka-Cray 1996, Baum et al. 1998, Bush et al. 1999). No presente estudo, em três das dez granjas amostradas foi possível isolar Salmonella a partir de fezes de lote. Duas destas granjas apresentaram acima de $50 \%$ das amostras colhidas positivas, indicando um alto nível de contaminação.

Nas granjas positivas, 8 sorotipos de Salmonella foram identificados, sendo o sorotipo Agona o mais freqüiente (Quadro 1). Este sorotipo tem sido um dos mais isolados em granjas de suínos, juntamente com os sorotipos Derby e Typhimurium (Fedorka-Cray 1996). Em alguns casos mais de um sorotipo foram encontrados numa mesma amostra, demonstrando a possibilidade de infecção múltipla em um mesmo lote de animais. Vários estudos apontam uma ampla variedade de sorotipos de Salmonella isolados nas propriedades de criação de suínos, indicando que existem diversas fontes de introdução do agente nas propriedades (Carlson \& Blaha 1998, 1999). Dentre essas fontes, a ração tem sido apontada como uma das principais formas de entrada do microrganismo nas propriedades produtoras de suínos e um dos maiores fatores de risco de contaminação dos plantéis (Fialho et al. 1985, Tielen et al. 1997, Beloeil et al. 1999). No presente estudo não houve isolamento de Salmonella a partir das amostras de ração colhidas na etapa de seleção inicial. Isto pode ter ocorrido, porque outras fontes de contaminação estavam envolvidas ou porque a ração não apresentava um nível de contaminação possível de ser detectado por métodos microbiológicos convencionais.

A primeira colheita realizada resultou no isolamento de Salmonella em todas as granjas escolhidas, ou seja, foram encontrados animais portadores mesmo nas granjas negativas no exame de fezes de lote. Cabe ressaltar que os animais amostrados nesta fase pertenciam ao mesmo grupo alojado no momento em que realizou-se a colheita de fezes de lote na granja (Quadro 2).

A excreção de Salmonella pelos suínos portadores ocorre de forma intermitente (Fedorka-Cray 1996), o que poderia explicar a discrepância entre os resultados das fezes de lote e da colheita individual dos mesmos animais. Neste sentido, tem sido observado que a repetição de amostragens numa mesma propriedade leva ao aumento do número de animais positivos detectados (Carlson \& Blaha 1998).

Quadro 1. Distribuição de sorotipos de Salmonella em fezes de lote de três granjas de terminação de suínos do Rio Grande do Sul

\begin{tabular}{cccc}
\hline Sorotipos & \multicolumn{3}{c}{ Granjas } \\
\cline { 2 - 4 } & P1 & P2 & P3 \\
\hline Agona & 7 & - & - \\
Bredeney & 1 & 2 & - \\
Derby & - & - & 1 \\
Panama & 1 & 2 & - \\
Salmonella sp & - & 7 & - \\
Total de isolamentos & 9 & 11 & 1
\end{tabular}

Quadro 2. Número de animais positivos e distribuição dos sorotipos de Salmonella isolados de fezes colhidas de suínos de três granjas no Rio Grande do Sul

\begin{tabular}{cccc}
\hline Colheita & \multicolumn{3}{c}{ Granjas } \\
\cline { 2 - 4 } & $\mathrm{N} 1$ & $\mathrm{~N} 2$ & $\mathrm{P} 1$ \\
\hline \multirow{2}{*}{ Primeira } & $\begin{array}{c}4^{\mathrm{a}} \text { (Agona, } \\
\text { Panama, } \\
\text { Salmonella } \mathrm{sp})\end{array}$ & $\begin{array}{c}2 \text { (Salmonella sp, } \\
\text { Schwarzengrund) }\end{array}$ & 1 (Agona) \\
Segunda & 0 & $\mathrm{NC}^{\mathrm{b}}$ & 1 (Mbandaka)
\end{tabular}

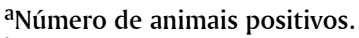

${ }^{\mathrm{b}} \mathrm{NC}=$ Não colhido.

Por outro lado, não é possível descartar que os animais tenham sido contaminados durante a permanência na granja, após a colheita das fezes de lote. Esta contaminação poderia ter ocorrido pelo contato com o ambiente ou consumo de lote de ração contaminada. Porém, também nas amostras de ração colhidas nesta etapa do trabalho não foi possível encontrar Salmonella, o que não descarta a possibilidade de lotes de ração anteriores à colheita terem sido contaminados.

Na segunda colheita realizada, após o repovoamento, observou-se que a granja $\mathrm{N} 1$, onde havia sido encontrado $\mathrm{o}$ maior número de animais excretores na primeira visita, não apresentava animais positivos. Por outro lado, a granja P1 continuava apresentando a presença de Salmonella nos suínos de terminação, indicando a permanência do agente na propriedade (Quadro 2). Estas duas situações parecem refletir o conceito de que são múltiplas as fontes de entrada da Salmonella na propriedade e longa a sua permanência no ambiente, caso não sejam tomadas medidas de manejo adequadas. $\mathrm{O}$ controle da presença de Salmonella nas granjas de terminação de suínos tem sido bastante discutido, havendo, entretanto, um consenso que o mesmo deve ser direcionado para a manutenção de baixos níveis de infecção e não para a erradicação do agente (Tiele et al. 1997, Davies \& Funk 1999). Os fatores citados como importantes para o controle tem sido o estabelecimento de um programa de desinfeção eficiente associado ao vazio sanitário e o controle da ração (Tielen et al. 1997). Entretanto, ainda é necessário determinar quais fatores de risco são importantes para as condições de manejo em nosso país, para que recomendações de controle sejam estabelecidas.

As colheitas realizadas no mesmo grupo de animais no matadouro resultaram na ausência de isolamento de Salmonella a partir de swabs retais. Estudos iniciais da excreção deste microrganismo por suínos basearam-se na realização de swabs retais de animais transportados ao matadouro (William \& Newell 1970). Desta forma, optou-se por este tipo de colheita, até mesmo pela facilidade em obter o material no abatedouro. Os primeiros resultados encontrados nas granjas $\mathrm{N} 1$ e P1 deixaram dúvidas a respeito deste método, o que determinou que fossem introduzidas as colheitas de conteúdo intestinal. A partir daí, a colheita de conteúdo intestinal realizada nos animais dos lotes subseqüientes amostrados resultou na deteç̧ão de animais excretores no momento do 
abate e que seriam considerados negativos pelo exame do swab retal.

Esta observação está de acordo com estudos mais recentes que concluíram que a colheita de swab retal é um método eficiente para o isolamento de Salmonella a partir de casos clínicos. Para a deteç̧ão de animais portadores, onde o título de bactérias excretadas nas fezes é geralmente baixo, este método torna-se pouco sensível (Hurd et al. 1999). Nielsen \& Baggesen (1997) afirmaram que o aumento na quantidade de fezes semeadas nos meios de cultura tem relação direta com a sensibilidade no isolamento de Salmonella sp.

Comparando os resultados de isolamento a partir de linfonodos mesentéricos e conteúdo intestinal, observa-se que há uma correspondência com os resultados das fezes colhidas na granja. Das amostras de fezes colhidas na granja $6,4 \%$ foram positivas, enquanto de $5,3 \%$ dos conteúdos intestinais e 5,6\% dos linfonodos foi possível isolar Salmonella. O lote com maior número de animais positivos na granja correspondeu àquele em que havia o maior número de animais com isolamento no abatedouro.

Já a observação dos isolamentos obtidos a partir de linfonodos e conteúdo intestinal de um mesmo animal demonstra que nem sempre há concordância entre ambos (Quadro 3). Isto está relacionado ao fato de que suínos infectados na granja podem tornar-se portadores em linfonodos e passarem a excretar de forma intermitente o agente. Esta excreção tenderia a aumentar em situações de estresse, como aquelas verificadas durante o transporte dos animais ao abatedouro. Por outro lado, $o$ transporte destes animais com outros não infectados pode resultar na infecção destes últimos, aumentando o número de animais positivos nas fezes. Tanto a presença de Salmonella nos linfonodos como nas fezes dos animais abatidos constituem risco para a contaminação cruzada das carcaças na linha de abate, sendo, por isto, $o$ número de portadores de Salmonella nos lotes considerado o principal ponto de controle da contaminação do produto final (Davies \& Funk 1999).

Nas diversas colheitas realizadas observou-se uma variação dos sorotipos isolados tanto entre granjas, como nas colheitas realizadas nos mesmos animais, confirmando que pode haver na propriedade infecção concomitante por diferentes sorotipos de Salmonella sp. Segundo Carlson \& Blaha (1999), esta situação é freqüentemente encontrada nas propriedades onde existem suínos portadores, sendo diferentes sorotipos detectados no ambiente, fezes e linfonodos dos animais.

Nas propriedades onde foram realizadas as colheitas 0 sorotipo Agona foi o mais encontrado, não tendo sido identificado o sorotipo Typhimurium, predominante na maioria dos estudos realizados em suínos. $O$ perfil de sorotipos isolados costuma variar em função do local de amostragem e ao longo do tempo. Desta forma, é impossível prever qual sorotipo terá maior importância em termos de ocorrência e como agente de infeç̧ões em animais e humanos (Davies \& Funk 1999).

Em relação à ocorrência de episódios de toxinfecção alimentar, os sorotipos Typhimurium, Enteritidis, Infantis, Agona e Heidelberg têm sido classificados como os mais freqüentes
Quadro 3. Isolamento de Salmonella de swabs retais, conteúdo intestinal e linfonodos provenientes dos mesmos animais

\begin{tabular}{|c|c|c|c|c|}
\hline Propriedade $^{a}$ & Animal & Swab retal & $\begin{array}{l}\text { Conteúdo } \\
\text { intestinal }\end{array}$ & Linfonodos \\
\hline & $\begin{array}{l}1 \\
2\end{array}$ & $\begin{array}{l}\text { Neg. } \\
\text { Neg. }\end{array}$ & $\begin{array}{l}\mathrm{NC}^{\mathrm{d}} \\
\mathrm{NC}\end{array}$ & $\begin{array}{l}\text { London } \\
\text { Agona }\end{array}$ \\
\hline $\mathrm{N} 1^{\mathrm{b}}$ & $\begin{array}{c}3 \\
4 \\
5-25\end{array}$ & $\begin{array}{l}\text { Neg. } \\
\text { Neg. } \\
\text { Neg. }\end{array}$ & $\begin{array}{l}\mathrm{NC} \\
\mathrm{NC} \\
\mathrm{NC}\end{array}$ & $\begin{array}{c}\text { Tenessee } \\
\text { London } \\
\text { Neg. }\end{array}$ \\
\hline $\mathrm{N} 2^{\mathrm{b}}$ & $\begin{array}{c}1 \\
2 \\
3-25 \\
1\end{array}$ & $\begin{array}{l}\text { Neg. } \\
\text { Neg. } \\
\text { Neg. } \\
\text { Neg. }\end{array}$ & $\begin{array}{l}\text { Lexington } \\
\text { Lexington } \\
\text { Neg. } \\
\text { NC }\end{array}$ & $\begin{array}{l}\text { Neg. } \\
\text { Neg. } \\
\text { Neg. } \\
\text { Bredeney }\end{array}$ \\
\hline $\mathrm{P} 1^{\mathrm{b}}$ & $\begin{array}{c}2 \\
3-25\end{array}$ & $\begin{array}{l}\text { Neg. } \\
\text { Neg. }\end{array}$ & $\begin{array}{l}\mathrm{NC} \\
\mathrm{NC}\end{array}$ & $\begin{array}{c}\text { Bredeney } \\
\text { Neg. }\end{array}$ \\
\hline $\mathrm{N} 1^{\mathrm{c}}$ & $\begin{array}{c}1-25 \\
1\end{array}$ & $\begin{array}{l}\text { Neg. } \\
\text { Neg. }\end{array}$ & $\begin{array}{c}\text { Neg. } \\
\text { London }\end{array}$ & $\begin{array}{l}\text { Neg. } \\
\text { Neg. }\end{array}$ \\
\hline $\mathrm{P} 1^{\mathrm{c}}$ & $\begin{array}{c}2 \\
3 \\
4-25\end{array}$ & $\begin{array}{l}\text { Neg. } \\
\text { Neg } \\
\text { Neg. }\end{array}$ & $\begin{array}{c}\text { Salmonella sp } \\
\text { Neg. } \\
\text { Neg. }\end{array}$ & $\begin{array}{l}\text { Neg. } \\
\text { Agona } \\
\text { Neg. }\end{array}$ \\
\hline
\end{tabular}

aNegativas (N1, N2) ou positiva (P1) na seleção inicial.

brimeira colheita.

'Segunda colheita.

${ }^{\mathrm{d}} \mathrm{NC}=$ Não colhido.

em diferentes países (Davies \& Funk 1999). No Brasil, os sorotipos Enteritidis, Typhimurium e Agona têm sido os mais encontrados (Gelli et al. 1998).

Um outro fator monitorado nas amostras de Salmonella sp é o perfil de resistência a antimicrobianos, principalmente após o surgimento de linhagens multi-resistentes no sorotipo Typhimurium (Wray et al. 1997).

No presente estudo, as amostras de Salmonella testadas apresentaram alta resistência apenas à sulfonamida $(97,8 \%) \mathrm{e}$ estreptomicina $(82,6 \%)$ (Fig. 1). Estes resultados estão de acordo com outros estudos, onde também o maior percentual de

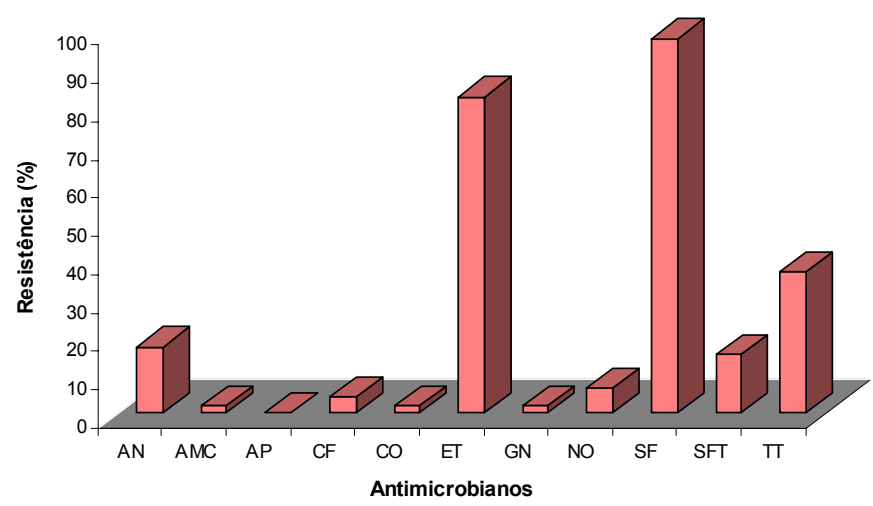

Fig. 1. Porcentagem de amostras resistentes de Salmonella sp isoladas em três propriedades do Estado do Rio Grande do Sul e testadas frente a diferentes antimicrobianos. $\mathrm{AN}=$ ácido nalidíxico, $\mathrm{AMC}=$ amicacina/ácido clavulânico, $\mathrm{AP}=$ ampicilina, $\mathrm{CF}=$ cefalotina, $\mathrm{CO}=$ cloranfenicol, ET = estreptomicina, $\mathrm{GN}=$ gentamicina, $\mathrm{NO}=$ neomicina, $\mathrm{SF}=$ sulfonamida, $\mathrm{SFT}=$ sulfonamida/trimetoprim, TT $=$ tetraciclina. 
resistência de diferentes sorotipos de Salmonella foi contra estes antimicrobianos (Wray et al. 1997, Fedorka-Cray et al. 1998, Bahson \& Fedorka-Cray 1999). Quanto à multi-resistência, apenas 7 amostras foram resistentes a mais de cinco antimicrobianos. Todas estas amostras foram isoladas de fezes colhidas das granjas N1 e N2 e pertenciam aos sorotipos Agona (2), Panama (2), Schwartzengrund (1), Lexington (1) e Salmonella sp (1).

Agradecimentos.- Às Instituições FAPERGS, CNPq e CAPES pelo auxílio financeiro. À Dra. Eliane Falavina dos Reis, Depto Bacteriologia, Instituto Oswaldo Cruz, pela sorotipagem das amostras.

\section{REFERÊNCIAS}

Alves J.C., Lázaro N.S. \& Hofer E. 1994. Salmonella sp. em linfonodos de suínos normais abatidos no estado do Rio de Janeiro. Revta Bras. Med. Vet. 16 (4):172-176.

Bahson B.P. \& Fedorka-Cray P.J. 1999. The association of antimicrobial resistance pattern and reported usage of antimicrobials in comercial growing pig production, p. 240-241. In: 3rd Symposium on the Epidemiology and Control of Salmonella in Pork, Washington DC, USA.

Barcellos D.E.S.N., Guizzardi I.I. \& Fallavena L.C.B. 1980. Freqüência e causa de diarréias bacterianas nas zonas criatórias do Vale do Taquari e Missões, RS. Boletim IPVDF, Porto Alegre, 7:27-37.

Barcellos D.E.S.N., Rodrigues N.C., Migliavacca F., Oliveira S.J. \& Borowski S.M. 1984. Ocorrência da salmonelose septicêmica em suínos no período neonatal, p. 29. In: I Congresso Nacional de Veterinários Especialistas em Suínos, Curitiba, PR.

Barry AL.\& Thornsberry C. 1985 . Susceptibility tests: Diffusion test procedures, p. 978-977. In: Lennette E.H., Balows A., Hausler W.J. \& Shadomy H.J. (ed.) Manual of Clinical Microbiology. 4th ed., ASM, Washington DC, USA.

Baum D.H., Harris D.L., Nielsen B. \& Fedorka-Cray P.J. 1998. Epidemiologic studies of Salmonella in swine using culture and ELISA, p. 75. In: 15th IPVS Congress, Birmingham, Inglaterra.

Beloeil P.A., Eveno E., Gerault P., Fravalo P., Rose V. \& Madec F. 1999. An exploratory study about contamination of pens of finishing pigs by ubiquitous Salmonella, p. 101-105. In: 3rd Symposium on the Epidemiology and Control of Salmonella in Pork, Washington DC, USA.

Berends B.R., Urlings J.M.A. \& Snijders F. 1996. Identification and quantification of risk factors in animal manegement and transport regarding Salmonella spp. in pigs. Int. J. Food Microbiol. 30:37-53.

Bush E.J., Wagner B. \& Fedorka-Cray P.J. 1999. Risk factors associated with shedding of Salmonella by U.S. finishing hogs, p. 141-152. In: 3rd Symposium on the Epidemiology and Control of Salmonella in Pork, Washington DC, USA.

Carlson A.R. \& Blaha T. 1998. On farm Salmonella control procedures - What is known? Investigations into zoonotic Salmonella in Minnesota, p. 141-152. In: Swine Disease Conference for Swine Practitioners, Ames, USA.

Carlson A.R. \& Blaha T. 1999. Investigation into the infection-contaminationinfection cycle of zoonotic Salmonella on swine farms: investigation of the simultaneous occurrence os Salmonella in animals and their environment on two selected Minnesota swine farms, p. 119-122. In: 3rd Symposium on the Epidemiology and Control of Salmonella in Pork, Washington DC, USA.
Costa G.A., Hofer E., Costa M., Silva J., Santos J. \& Doria J. 1972. Sobre o isolamento de salmonelas de gânglios linfáticos de suínos abatidos no matadouro da cidade de Salvador, Bahia. Mem. Inst. Oswaldo Cruz 70:417-431.

Davies P.R. \& Funk J.A. 1999. Epidemiology and control of Salmonella in pork - some of questions, p. 1-11. In: 3rd Symposium on the Epidemiology and Control of Salmonella in Pork, Washington DC, USA.

Fedorka-Cray P.J. 1996. The connection between Salmonella, swine, and food safety, p. 25-45. In: George A. Young Conference, USA.

Fedorka-Cray P.J. \& Gray J.T. 1996. Current state of Salmonella in swine, p. 1526. In: The Allen D. Leman Swine Conference, Minnesota, USA.

Fedorka-Cray P.J., Dagartz L.A., Thomas L.A. \& Gray J.T. 1998. Survey of Salmonella serotypes in feedlot cattle. J. Food Prot. 61:525-530.

Ferraz I.B.F., Reis R., Carvalho C.M.M.P., Figueiredo F.A.M. \& Nascimento, E.F. 1984. Aspectos clínicos e bacteriológicos da forma enterocolítica da salmonelose suína em Minas Gerais, p. 30-31. In: I Congresso Nacional de Veterinários Especialistas em Suínos, Curitiba, PR.

Fialho E.T., Sobestiansky J., Brito J.R.F., Bellaver C. \& Wentz, I. 1985. Composição química e ocorrência de Salmonella em alimentos e concentrados utilizados em ração de suínos. Pesq. Agropec. Bras. 20(3):377-384.

Gelli D.S., Jakabi M. \& Sakata H. 1998. Salmonelas isoladas de alimentos no período de 1985-1996 no estado de São Paulo, Brasil, p. 105. In: V Congresso Latino-Americano de Microbiologia e Higiene de Alimentos, Águas de Lindóia, SP.

Hirsh D.C. 1990. Salmonella, p.110-115. In: Biberstein E.L. \& Zee Y.C. (ed.) Review of Veterinary Microbiology. Blackwell, Orlando, USA.

Hurd H.S., Schlosser W.D. \& Ebel E.D. 1999. The effect of intermittent shedding on prevalence estimation in populations, p. 57-62. In: 3rd Symposium on the Epidemiology and Control of Salmonella in Pork, Washington DC, USA.

Langenegger C.H. \& Langenegger J. 1975. Surtos de salmonelose por Salmonella typhisuis em suínos no estado do Rio de Janeiro. Pesq. Agropec. Bras., Sér. Vet., 10:111-114.

Langenegger C.H., Alfinito J. \& Langenegger J. 1983. Salmonelas isoladas de suínos de abate do estado do Pará. Pesq. Vet. Bras. 3(3):91-94.

Nielsen B. \& Baggesen D.L. 1997. Update on laboratory diagnosis of subclinical Salmonella infection in pigs, p. 19-25. In: 2nd Symposium on the Epidemiology and Control of Salmonella in Pork, Copenhagen, Dinamarca.

Neiva C. 1946. Incidência de salmonelas em suínos, p. 430-435. In: 3ํㅡongresso Brasileiro de Veterinária, Porto Alegre, RS.

Tielen M.J.M., Van Schie F.W., Van der Wolf P.J., Elbers A.R.J., Koppens J.M.C.C. \& Wolbers W.B. 1997. Risk factors and control measures for subclinical Salmonella infection of pig herds, p. 32-35. In: 2nd Symposium on the Epidemiology and Control of Salmonella in Pork, Copenhagen, Dinamarca.

Wilcock B.P. \& Schwartz K.J. 1993. Salmonellosis, p. 570-583. In: Leman, A.D., Straw B.E., Mengeling W.L., D’Allaire S. \& Taylor D.J. (ed.) Diseases of Swine. 7th ed. lowa State University, Ames, USA.

Williams L.P. \& Newell K.W. 1970. Salmonella excretion in joy-riding pig. Am. J. Public Health 60(5):926-929.

Wray C.W. \& Sojka W.J. 1977. Reviews of the progress of dairy science: bovine salmonellosis. J. Dairy Sci. 44:383-425.

Wray C. Davies R.H., Jones Y. \& Chappel S. 1997. Antibiotic resistance and Salmonella infection in pigs in Great Britain, p. 247-250. In: 2nd Symposium on the Epidemiology and Control of Salmonella in Pork, Copenhagen, Dinamarca. 